Title:

\title{
Implementation and Evaluation of Simultaneous Video-Electroencephalography (Video-EEG) and functional Magnetic Resonance Imaging (fMRI)
}

Authors:

Umair J Chaudhary ${ }^{1,3}$, MBBS, MSc, Vasileios Kokkinos ${ }^{1,3}$, MSc, David W Carmichael ${ }^{1,3}$, PhD, MInstP, Roman Rodionov ${ }^{1,3}, \mathrm{PhD}$, David Gasston ${ }^{4}$, BSc, John S Duncan ${ }^{1,2,3}$, MA, DM, FRCP, FMedSci, Louis Lemieux ${ }^{1,3}, \mathrm{PhD}, \mathrm{CSci}$, MInstP

\section{Affiliations:}

${ }^{1}$ Department of Clinical and Experimental Epilepsy, UCL Institute of Neurology, University College London, Queen Square, London WC1N 3BG, UK

${ }^{2}$ National Hospital for Neurology and Neurosurgery, Queen Square, London WC1N 3BG, UK

${ }^{3}$ MRI Unit, National Society for Epilepsy, Chesham Lane, Chalfont St Peter, Buckinghamshire, SL9 0RJ, UK

${ }^{4}$ Section of Cognitive Neuropsychiatry, Institute of Psychiatry, Kings College London, London, UK

\section{Corresponding author:}

Prof. Louis Lemieux, Department of Clinical and Experimental Epilepsy, UCL Institute of Neurology, Queen Square, London WC1N 3BG, UK.

Email: 1.lemieux@ion.ucl.ac.uk., Tel.: +44-1494-601-361, Fax: +44-1494-875-666 


\begin{abstract}
$\underline{\text { Abstract }}$
The objective of this study is to demonstrate that the addition of video to EEG-correlated fMRI for simultaneous recording can be done efficiently. We investigated the effect of placing EEG, video equipment and their required power supplies inside the scanner room, on EEG, video and MRI data quality, and evaluated video-EEG-fMRI by modelling a hand motor task. Gradient-echo echo-planner images (EPI) were acquired on a 3T MRI scanner at variable camera positions in a test object (with and without radiofrequency amplification), and human subjects. EEG was recorded using a commercial MR-compatible 64-channel cap and amplifiers. Video recording was performed using a two-camera custom-made system with EEG synchronization. An in-house script was used to calculate signal to fluctuation noise ratio (SFNR) from raw EPI, and the results compared in data from human subjects with and without concurrent video recording. Five subjects were investigated with video-EEGfMRI while performing hand motor task. The fMRI time series data was analysed using statistical parametric mapping based on the paradigm-prescribed block design and on an alternative video based block design. Introduction of the cameras did not alter the SFNR substantially in the test object and human subjects. Video and EEG quality assessed by two expert observers also did not show any significant artefact. The SPM $\{\mathrm{T}\}$ maps from video based general linear models revealed additional BOLD responses in the expected locations for noncompliant subjects with the paradigm design. We conclude that video-EEG-fMRI set up can be implemented without affecting the data quality significantly and may provide valuable information on actual behaviour that may be used for the analysis of fMRI data.
\end{abstract}




\section{Introduction:}

The recording and analysis of ictal events on EEG have a central role in the correct diagnosis of epilepsy. In addition simple partial seizures may not have a definite signature on scalp recorded EEG. In this respect video-EEG recording has proven to be pivotal in localizing the epileptogenic zone and seizure propagation with a good diagnostic yield (1-3).

EEG-fMRI is an imaging technique involving the simultaneous recording of scalp EEG and fMRI data aimed mainly at capturing haemodynamic changes linked to specific features on the EEG. The simultaneous recording of the two types of signal (EEG and fMRI) with sufficient quality requires specific hardware and software solutions to avoid or reduce interference between the EEG recording system and MR scanner (4-7). In most applications, the fMRI data are analysed by building a general linear model (GLM) based on events of interest that are identified and classified on EEG. These events are convolved with the haemodynamic response function or other basis set along with temporal and dispersion derivatives to derive blood oxygen level dependent (BOLD) signal changes (8;9).The majority of EEG-fMRI studies have focussed on interictal epileptiform discharges (IED) which are generated by the irritative zone (10) and may be different from the ictal onset zone (11). This indicates the need to study the haemodynamic correlates of seizures, which has been investigated in a relatively small number of cases mainly incidentally during studies aimed at the study of interictal events $(9 ; 12-19)$. In comparison, the ictal events were triggered by experimental manipulation in a few cases of reflex epilepsy (16).

The analysis of this type of data to reveal ictal BOLD changes remains a challenge due to the potential complexity of the events and effects of head movement. A variety of strategies have been applied to identify seizures during EEG-fMRI data acquisition, such as; EEG as a marker of seizure onset and offset $(9 ; 13-17 ; 20-27)$, button pressing by the patient $(9 ; 26)$, di- 
rect observation of the patient $(15 ; 17)$, detection of an acoustic signal from the patient $(15)$, eye tracking system (28) and concurrent but non-synchronized video recording $(27 ; 29-31)$. However, these methods may be unreliable as markers of the ictal events particularly in the absence of a data synchronization mechanism (16).

We implemented synchronized video recording with EEG-fMRI as an attempt to combine the capability of video-EEG to detect and characterise ictal events with the localising power of fMRI. In particular, this should provide the opportunity to identify the onset and evolution of seizures semiologically and electrophysiologically (as for clinical video-EEG telemetry) allowing greater precision and specificity in building models for the analysis of the fMRI time series. We propose the use of two video cameras; one capturing facial semiology $(\mathrm{C} 1$ : scanner bore mounted detachable camera), and a second one monitoring limb and body movements (C2: wall-mounted non-detachable camera inside the scanner room). We investigated the compatibility of dual video recording synchronised with EEG-fMRI equipment inside a 3 Tesla MR scanner based on the evaluation of possible noise production in EEG, video and MR data quality secondary to the introduction of the video recording equipment in the EEGfMRI data acquisition set up. We also evaluated video-EEG-fMRI in a hand motor paradigm to utilize supplementary information from video in fMRI data analysis. 


\section{Materials and Methods:}

This study was conducted at the MRI Unit, National Society for Epilepsy, Chalfont St Peter, UK. The effect of video cameras on EEG-fMRI data quality was assessed in a test object (17 cm diameter spherical plastic vessel filled with a doped agar gel, Dielectric, Inc., Madison, WI, USA) and human subjects. The test object was scanned with variable camera position (C1 at positions P1: edge of scanner bore and P2: inside the scanner bore and $\mathrm{C} 2$ switched on (S1)) and without cameras (C1 at P0: removed from the scanner room and C2 switched off (S0)).

EEG and fMRI data was acquired with video cameras in place (C1 at P2 and C2 at S1) in 12 subjects, and without video cameras in 12 subjects ( $\mathrm{C} 1$ at $\mathrm{P} 0$ and $\mathrm{C} 2$ at $\mathrm{S} 0$ ), from August 2008 to April 2009. Subjects gave informed written consent in accordance with the requirements of the Joint Research Ethics Committee of the National Hospital for Neurology and Neurosurgery (UCLH NHS Trust) and UCL Institute of Neurology, Queen Square, London, UK.

\section{MRI Acquisition:}

Subjects were fitted with an EEG cap (see below), ear plugs and their head immobilised using a vacuum cushion. They were asked to remain still with eyes closed. Images were acquired using a 3T GE Signa ${ }^{\circledR}$ Excite HDX echospeed MRI scanner (GE, Milwaukee, USA) with a standard transmit/receive head coil. Five minute sessions of gradient echo T2*-weighted single-shot echo-planar images (EPI) were performed with radiofrequency (RF) amplifier switched on and off for test object. Twenty minute EPI sessions were performed for human subjects with RF amplifier switched on. Image parameters were as follows: TE $=30 \mathrm{~ms}$, TR $=3000 \mathrm{~ms}=$ Flip angle $=90^{\circ}, 442.4 \mathrm{~mm}$-thick slices with $0.6 \mathrm{~mm}$ gap; FOV $=24 \times 24 \mathrm{~cm}^{2}$, matrix: 64x64. 


\section{EEG Acquisition and Processing:}

In human subjects, scalp EEG was recorded during MR scanning using a 64 channel MRcompatible electrode cap (BrainCap MR, Easycap, Herrsching-Breitbrunn, Germany), according to the extended international 10-20 system. Signals were amplified by $2 \times 32$ MRcompatible amplifiers (BrainAmp MR plus, Brain Products, Munich, Germany) with a gain of 1500 , band-pass filter settings: $0.016 \mathrm{~Hz}-1 \mathrm{kHz}, 16-\mathrm{bit}$ digitization $(0.5 \mathrm{uV}$ resolution) and sampling rate of $5 \mathrm{kHz}$. EEG data was transmitted from the amplifiers to the recording computer located in the control room via fibre-optic cable. EEG and MR scanner clocks were synchronized. Two additional electrodes were used to record electroocculogram (EOG) and electrocardiogram (ECG). Electrode impedance was kept below 10kOhms.

\section{Video Monitoring and Recording:}

Two cameras were used to monitor and record the subject's behaviour during MR scanning (schematic representation in Figure 1). An MR-compatible camera (MR-Cam 12M, MRC Systems GmbH, Heidelberg, Germany) (C1) was positioned inside/around the bore of the scanner focusing on the subject's face, recording facial expressions and head movements. The video signals from $\mathrm{C} 1$ were transmitted to the recording computer placed outside the scanner room through shielded cable and low-pass filter (LPF) (1MHz) (MRC Systems

GmbH, Heidelberg, Germany) to provide RF shielding. The LPF box was also grounded to the scanner room shield.

A second video camera (Handicam DCR-HC51E, Sony Corporation, San Diego, CA, USA) (C2) was positioned on the wall facing the scanner bore allowing a view of the subject's entire body. The video signals from $\mathrm{C} 2$ to the recording computer were transmitted using a custom-built filter system consisting of electric power supply sockets for camera and optical fire

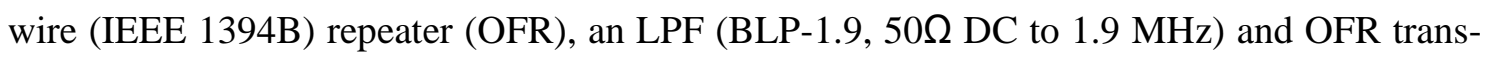


mitter box enclosed in an aluminium box. Both $\mathrm{C} 2$ and filter box were permanently attached at a safe distance of $3 \mathrm{~m}$ and a height of $2.5 \mathrm{~m}$ in front of the scanner.

The video output signals from $\mathrm{C} 1$ and $\mathrm{C} 2$ were different being analogue and digital respectively. An ADVC110-High-Quality, Bi-Directional Analogue/Digital Video Converter (Thomson Worldwide, Cergy Pontoise Cedex, France) converted digital video signals from C2 to analogue video signals. Analogue video signals from both video cameras were fed into a video multiplexer (HA-402TX Quad Processor, Ultima Precision Co Ltd, Taiwan) for picture-in-picture video format. A ADVC110-high quality, bi-directional analogue/digital video converter (Thomson Worldwide, Cergy Pontoise Cedex, France) converted the analogue to digital video signals for display and input to the recording computer. The video images were recorded synchronously with the EEG (Brain Vision Recorder, Brain Products, Munich, Germany). The above-described video input system has a maximum transmission delay of $\approx$ $150 \mathrm{~ms}$ as specified by the manufacturers.

\section{Experimental Design for Hand Motor Task}

Five subjects undergoing video-EEG-fMRI were asked to perform repetitive finger thumb opposition task starting with left hand, followed by right hand and then rest, each lasting 30 seconds over a period of 5 minutes. The subjects were instructed to focus on the head coil mirror through which they could see the radiographers in control room and follow the cue for changing hands and rest.

\section{Data Analysis: Image, EEG and Video Quality}

We calculated the signal to fluctuation noise ratio (SFNR) to evaluate MR data quality in relation to the presence of video recording. The SFNR was defined as mean signal intensity of a voxel divided by temporal standard deviation of the same voxel. The region of interest was 
defined as the central $11 \times 11 \times 11$ voxels (Figure $2 \mathrm{~A}$ ) of the volume which were always selected automatically presuming the phantom / subject head is at the centre of the field of view during image acquisition. At a second step multiple SFNR values were calculated by dividing the mean signal intensity by the temporal standard deviation of the difference between two adjacent volumes. The SFNR in raw EPI were calculated using an in house script written in Matlab version 6.5 R13 (MathWorks, Natick, MA, U.S.A.) and Statistical Parametric Mapping2 (SPM2) software (available from: http://www.fil.ion.ucl.ac.uk/spm) running on a Dell Inspiron 1525 under Red Hat Linux 9. The script also displayed standard deviation (SD) summary maps and created movies of raw EPI with RF amplifier switched off for review. We used Mann-Whitney test (SPSS version 13) to compare SFNR from EPI acquired at variable camera positions in the test object, as well as from EPI acquired with and without video recording in human subjects. EEG data was corrected for scanning and pulse artefacts $(4 ; 5)$, and synchronized video-EEG was reviewed visually by two expert observers using Brain Vision Analyzer (BVA) software version 2 (Brain Products GmbH, Munich, Germany).

\section{Hand Motor Task}

The fMRI time series data was analysed using SPM5. The EPI time series were realigned, normalized and spatially smoothed with a cubic Gaussian Kernel of $8 \mathrm{~mm}$ full width at half maximum. Two general linear models (GLM) were built to determine the presence of experimental condition related BOLD effects. A first GLM was built based on the specified block design with a canonical basis set. A second GLM was built based on review of the video to identify left and right hand finger tapping blocks, convolved with canonical basis set. Scan realignment parameters from image pre-processing were included as confounds in both the GLMs and SPM $\{\mathrm{T}\}$ maps were generated (corrected for family wise error: thresholded at $\mathrm{p}<0.05$, uncorrected: thresholded at $\mathrm{p}<0.001$ ). 


\section{Results:}

Visual inspection of the movie of raw EPI image time series with RF amplifier switched off did not reveal any artefact, spike noise or electrostatic interference, and comparison of SD maps of the time series with and without RF amplification in test object did not reveal any RF interference for any of the camera configurations (Figure 2A). The difference between SFNR estimates for cameras at variable positions (P1, P2, S1, P0 and S0) was not statistically significant (Figure $2 \mathrm{~B}$ ).

The SFNR for 12 subjects without the camera ranged from 26.7 to 73.9 with a mean of 50.9 Comment [LL1]: U se words like stable to describe results in the discussion; stick to giving numbers in the results section. (95\% CI; 42.5 - 59.3), and SFNR for 12 subjects with camera ranged from 33.7 to 75.7 with a mean of 49.5 (95\% CI; 42.9- 56.2). The comparison of SFNR from raw EPI of human subjects with / without camera showed no significant difference $(p>0.05)$ (Figure 2C).

Visual inspection and comparison of the video and EEG inside and outside the MR scanner did not reveal any RF or electromagnetic interference artefact as assessed by expert observers with experience in EEG-fMRI (Figure 3A).

BOLD activations were observed in expected hand motor areas which were concordant for both analysis strategies in $4 / 5$ subjects. Review of video revealed that subject 4 performed the task randomly alternating between right and left hand. The paradigm-based block design analysis did not reveal any significant BOLD response. Incorporation of video-derived information in the second GLM showed significant BOLD activations in the motor cortex [Left: Cluster Size $=18$, Voxel $\mathrm{t}=5.7(\mathrm{Z}$ equivalent $=5.2)$, Right: $[$ Cluster Size $=18$, Voxel $\mathrm{t}$ $=5.8(\mathrm{Z}$ equivalent $=5.2)]$ and supplementary motor cortex $($ SMA $)[$ Cluster Size $=98$, Voxel $t=6(Z$ equivalent $=5.5)]$. By lowering the threshold, SPM $\{T\}$ maps (uncorrected for FWE) for paradigm-based block design revealed BOLD activations around motor cortex [Right: Cluster Size $=509$, Voxel $\mathrm{t}=4.87(\mathrm{Z}$ equivalent $=4.57)$, Left: Cluster Size $=178$, Voxel $\mathrm{t}=$ 
$4.15(\mathrm{Z}$ equivalent $=3.95)]$ and SMA $[$ Cluster Size $=136$, Voxel $t=4.36(\mathrm{Z}$ equivalent $=$ 4.14)]. In comparison, $\operatorname{SPM}\{\mathrm{T}\}$ maps for the second video based GLM showed BOLD response in hand motor cortex [Right: Cluster Size $=689$, Voxel $t=4.52(\mathrm{Z}$ equivalent $=4.26)$, Left: Cluster Size $=764$, Voxel $t=5.7(Z$ equivalent $=5.2)]$, SMA $[$ Cluster Size $=4587$, Voxel $\mathrm{t}=6(\mathrm{Z}$ equivalent $=5.52)]$ and pre-motor cortex $[$ Right: Cluster Size $=4587$, Voxel $\mathrm{t}$ $=5.78(Z$ equivalent $=5.29)$, Left: Cluster Size $=523$, Voxel $t=5.13(Z$ equivalent $=4.77)]$ (Figure 3B). 


\section{$\underline{\text { Discussion and Conclusion }}$}

We evaluated the effect of using two powered video cameras on simultaneously acquired EEG and fMRI data quality. Our results demonstrate that there was no significant deterioration of the data quality and therefore simultaneous video-EEG-fMRI was implemented successfully.

Generally, in-built and standard camera systems provided by MR manufacturers have limitations including low video quality/resolution and limited view of the patient's body (32). Though video recording has been employed previously to record patient's behaviours during EEG-fMRI data acquisition (27;29-31), but to the best of our knowledge there has been no report on the effect of this on the image quality. Moreover, the video recordings were not synchronized with the EEG thus raising the issue of possible delay between video and EEG signals. Hence, the information obtained from video to be included in the design matrix for fMRI data analysis may become unreliable specially on the temporal scale. The video component of the video-EEG-fMRI system proposed in this report is compatible with the EEG recording system, thus providing synchronised video-EEG which is in turn synchronized with the MR scanner clock and scan acquisition. In this manner, EEG sampling remains constant relative to the scanner gradient switching and facilitates (33) the removal of imaging (Brain Vision Analyzer, Brain Products, Munich, Germany) and pulse artefact (4;5). Furthermore, the video recording system enables us to record two different views of the patient. One camera records the subject's facial expressions and head movement and the second camera records body and limb movements. The necessary equipment includes commercially available and custom made equipment and the set up can be assembled and disassembled in any MRI suite. In comparison to other camera systems established in an MRI scanner environment $(32 ; 34)$ the current system does not need any additional mirrors to reflect images of the patient or extra illumination of the subject. The image of the subject is recorded directly and the 
ambient scanner room and bore lighting provide sufficient luminescence to record video (Figure 3A).

The wall camera which is a commercially available camera provided a wider view of the patient's body. It was mounted at a sufficient distance (3 metres) from the scanner so that it does not interfere with the scanner's magnetic field which could result in artefacts and degraded image quality. An in-house built power and data line filter was used to reduce any electromagnetic interference from the power supply and data inputs of the wall camera. The conversion of digital electrical signals to optical signals was performed to ensure that electrical signals do not contribute to the noise production and eliminate the possibility of transmission of unwanted RF signals through the Faraday cage.

MR image quality assessment is routinely performed by calculating SNR (35) and specifically SFNR for functional time series $(36 ; 37)$. The bore camera was tested by the manufacturers (MR-Cam 12M, MRC Systems GmbH, Heidelberg, Germany), in a Siemens 1.5 Tesla Symphony scanner using fast gradients sequences on a water phantom. The manufacturer calculated mean and SD of noise in the MR images to evaluate the interference caused by the camera and found that this remained stable (personal communication). In this study, we tested two cameras in a 3 Tesla MRI scanner with an EPI sequence on a test object and human subjects for video-EEG-fMRI. We did not observe any significant artefact production due to the video recording. The SFNR was not significantly altered by the video recording. Similarly video recording inside MR scanner was also free of any major artefact. Thus, video recording can be implemented without any significant decline in the data quality.

The information provided by the video recording may be used efficiently for modelling and data analysis in subjects not complying with the experiment design. This is evident from the results of the finger tapping motor paradigm whereby incorporation of information provided by video recording into the design matrix revealed additional clusters of BOLD activations in 
SMA and pre-motor cortex. These findings are concordant with previously published findings for the brain areas responsible for finger tapping (38-40). We propose that the additional information provided by video will specifically be very useful in the studies of epileptic events. In conclusion, the compatibility of video cameras with EEG-fMRI equipment was evaluated here, and no significant deterioration in image quality was detected in the fMR images or in the EEG quality. The incorporation of video will be useful for event related analyses of motor activity that is not amenable to block-design analyses and future studies will benefit from the opportunity to use video-EEG-fMRI for studying ictal and interictal epileptic events. 
Acknowledgements: This work was partly funded through a grant from the Medical Research Council (MRC grant number G0301067) and the Higher Education Commission of Pakistan. Equipment purchase was partly funded by UCL Institute of Neurology. This work was undertaken at UCLH/UCL who received a proportion of funding from the Department of Health's NIHR Biomedical Research Centres funding scheme. We are grateful to the Big Lottery Fund, Wolfson Trust and National Society for Epilepsy for supporting the NSE MRI scanner. Many thanks to Philippa Bartlett, Elaine Williams, Jane Burdett, Rachel Thornton, Serge Vulliemoz, Anna Vaudano and Christian Vollmar for their help with set up and MRI scanning. 


\section{Figure Legends:}

\section{Figure 1:}

\section{(A) Circuit Diagram (B) System Set up}

\section{Figure 2:}

(A) Display of single slices of echo planner images (EPI) in the test object at variable camera positions. Region of interest (ROI) is shown in the centre (marked red). Mean time courses for different acquisitions with radiofrequency (RF) amplifier on and off are also shown.

(B) Mean SFNR (95\% Confidence Interval) (CI) calculated from different volumes of EPI from a test object with RF amplifier on and off, at variable camera positions. The difference between SFNR at variable camera position was not statistically significant: [RF On; C1 @ P0, C2 @ S0 VS C1 @ P1, C2 @ S1: p=0.2, C1 @ P0, C2 @S0VSC1 @ P2, C2 @ S1: p=0.7, RF Off; C1 @P0, C2 @ S0 VS C1 @ P1, C2 @ S1: $p=0.1, \mathrm{C} 1 @ \mathrm{P} 0, \mathrm{C} 2$ @S0 VS C1 @ P2, C2 @ S1: $p=0.8] .(\mathrm{C} 1=$ Scanner bore mounted detachable camera, $\mathrm{C} 2=$ Wall-mounted camera inside the scanner room, $\mathrm{P} 0=\mathrm{C} 1$ removed from the scanner room, $\mathrm{P} 1=\mathrm{C} 1$ at the edge of scanner bore, $\mathrm{P} 2=\mathrm{C} 1$ inside the scanner bore, $\mathrm{S} 0=\mathrm{C} 2$ switched off, $\mathrm{S} 1=\mathrm{C} 2$ switched on).

(C) Mean SFNR with 95\% CI from EPI in human subjects (12 Subjects with and 12 subjects without camera). The difference between SFNR in two groups was not statistically significant $(p=0.6)$. 


\section{Figure 3:}

(A) Representative sample of Synchronized Video-EEG

(B) Subject 4: BOLD activations for left and right hand finger tapping overlaid on normalized EPI (i) paradigm specific block design analysis revealed BOLD responses around primary motor cortex in subject 4 (being non-compliant to the paradigm design). (ii) Incorporation of video information about timing of finger tapping into video based block design GLM, revealed additional BOLD responses in primary motor cortex, supplementary motor cortex and pre-motor cortex. 


\section{Reference List}

(1) Binnie CD, Rowan AJ, Overweg J, Meinardi H, Wisman T, Kamp A, et al. Telemetric EEG and video monitoring in epilepsy. Neurology 1981 Mar;31(3):298-303.

(2) Cascino GD. Video-EEG monitoring in adults. Epilepsia 2002;43 Suppl 3:80-93.

(3) Kaplan PW, Lesser RP. Noninvasive EEG. Baltimore: Williams \& Wilkins; 1996.

(4) Allen PJ, Polizzi G, Krakow K, Fish DR, Lemieux L. Identification of EEG events in the MR scanner: the problem of pulse artifact and a method for its subtraction. Neuroimage 1998 Oct;8(3):229-39.

(5) Allen PJ, Josephs O, Turner R. A method for removing imaging artifact from continuous EEG recorded during functional MRI. Neuroimage 2000 Aug;12(2):230-9.

(6) Krakow K, Allen PJ, Symms MR, Lemieux L, Josephs O, Fish DR. EEG recording during fMRI experiments: image quality. Hum Brain Mapp 2000 May;10(1):10-5.

(7) Lemieux L, Allen PJ, Franconi F, Symms MR, Fish DR. Recording of EEG during fMRI experiments: patient safety. Magn Reson Med 1997 Dec;38(6):943-52.

(8) Salek Haddadi A, Diehl B, Hamandi K, Merschhemke M, Liston A, Friston K, et al. Hemodynamic correlates of epileptiform discharges: an EEG-fMRI study of 63 patients with focal epilepsy. Brain Res 2006 May 9;1088(1):148-66.

(9) Salek-haddadi A, Lemieux L, Merschhemke M, Friston KJ, Duncan JS, Fish DR. Functional magnetic resonance imaging of human absence seizures. Ann Neurol 2003 May;53(5):663-7.

(10) Luders HO, Najm I, Nair D, Widdess-Walsh P, Bingman W. The epileptogenic zone: general principles. Epileptic Disord 2006 Aug;8 Suppl 2:S1-S9.

(11) Rosenow F, Luders H. Presurgical evaluation of epilepsy. Brain 2001 Sep;124(Pt 9):1683700 .

(12) Di Bonaventura C, Carnfi M, Vaudano AE, Pantano P, Garreffa G, Le PE, et al. Ictal hemodynamic changes in late-onset rasmussen encephalitis. Ann Neurol 2006 Feb;59(2):4323.

(13) Gotman J, Grova C, Bagshaw A, Kobayashi E, Aghakhani Y, Dubeau F. Generalized epileptic discharges show thalamocortical activation and suspension of the default state of the brain. Proc Natl Acad Sci U S A 2005 Oct 18;102(42):15236-40.

(14) Kobayashi E, Hawco CS, Grova C, Dubeau F, Gotman J. Widespread and intense BOLD changes during brief focal electrographic seizures. Neurology 2006 Apr 11;66(7):1049-55.

(15) Di Bonaventura C, Vaudano AE, Carni M, Pantano P, Nucciarelli V, Garreffa G, et al. EEG/fMRI study of ictal and interictal epileptic activity: methodological issues and future perspectives in clinical practice. Epilepsia 2006;47 Suppl 5:52-8.

(16) Salek Haddadi A, Mayer T, Hamandi K, Symms M, Josephs O, Fluegel D, et al. Imaging seizure activity: A combined EEG/EMG-fMRI study in reading epilepsy. Epilepsia 2008 Aug 19. 
(17) Tyvaert L, Hawco C, Kobayashi E, LeVan P, Dubeau F, Gotman J. Different structures involved during ictal and interictal epileptic activity in malformations of cortical development: an EEG-fMRI study. Brain 2008 Aug;131(Pt 8):2042-60.

(18) Liu Y, Yang T, Liao W, Yang X, Liu I, Yan B, et al. EEG-fMRI study of the ictal and interictal epileptic activity in patients with eyelid myoclonia with absences. Epilepsia 2008 Jul 24.

(19) Moeller F, Siebner HR, Wolff S, Muhle H, Granert O, Jansen O, et al. Simultaneous EEGfMRI in drug-naive children with newly diagnosed absence epilepsy. Epilepsia 2008 Sep;49(9):1510-9.

(20) Aghakhani Y, Bagshaw AP, Benar CG, Hawco C, Andermann F, Dubeau F, et al. fMRI activation during spike and wave discharges in idiopathic generalized epilepsy. Brain 2004 May;127(Pt 5):1127-44.

(21) Hamandi K, Salek-haddadi A, Laufs H, Liston A, Friston K, Fish DR, et al. EEG-fMRI of idiopathic and secondarily generalized epilepsies. Neuroimage 2006 Jul 15;31(4):1700-10.

(22) Hamandi K, Laufs H, Noth U, Carmichael DW, Duncan JS, Lemieux L. BOLD and perfusion changes during epileptic generalised spike wave activity. Neuroimage 2008 Jan 15;39(2):608-18.

(23) Iannetti GD, Di BC, Pantano P, Giallonardo AT, Romanelli PL, Bozzao L, et al. fMRI/EEG in paroxysmal activity elicited by elimination of central vision and fixation. Neurology 2002 Mar 26;58(6):976-9.

(24) Laufs H, Lengler U, Hamandi K, Kleinschmidt A, Krakow K. Linking generalized spikeand-wave discharges and resting state brain activity by using EEG/fMRI in a patient with absence seizures. Epilepsia 2006 Feb;47(2):444-8.

(25) LeVan P, Tyvaert L, Moeller F, Gotman J. Independent component analysis reveals dynamic ictal BOLD responses in EEG-fMRI data from focal epilepsy patients. Neuroimage 2009 Aug 6.

(26) Salek-haddadi A, Merschhemke M, Lemieux L, Fish DR. Simultaneous EEG-Correlated Ictal fMRI. Neuroimage 2002 May;16(1):32-40.

(27) Tyvaert L, LeVan P, Dubeau F, Gotman J. Noninvasive dynamic imaging of seizures in epileptic patients. Hum Brain Mapp 2009 Jun 8.

(28) Krakow K, Baxendale SA, Maguire EA, Krishnamoorthy ES, Lemieux L, Scott CA, et al. Fixation-off sensitivity as a model of continuous epileptiform discharges: electroencephalographic, neuropsychological and functional MRI findings. Epilepsy Res 2000 Nov;42(1):1-6.

(29) Archer JS, Waites AB, Abbott DF, Federico P, Jackson GD. Event-related fMRI of myoclonic jerks arising from dysplastic cortex. Epilepsia 2006 Sep;47(9):1487-92.

(30) Federico P, Abbott DF, Briellmann RS, Harvey AS, Jackson GD. Functional MRI of the pre-ictal state. Brain 2005 Aug;128(Pt 8):1811-7.

(31) Krings T, Topper R, Reinges MH, Foltys H, Spetzger U, Chiappa KH, et al. Hemodynamic changes in simple partial epilepsy: a functional MRI study. Neurology 2000 Jan 25;54(2):524-7. 
(32) Neuner I, Wegener P, Stoecker T, Kircher T, Schneider F, Shah NJ. Development and implementation of an MR-compatible whole body video system. Neurosci Lett 2007 Jun $13 ; 420(2): 122-7$.

(33) Mandelkow H, Halder P, Boesiger P, Brandeis D. Synchronization facilitates removal of MRI artefacts from concurrent EEG recordings and increases usable bandwidth. Neuroimage 2006 Sep;32(3):1120-6.

(34) Wild B, Erb M, Lemke N, Scholz P, Bartels M, Grodd W. Video camera and light system for application in magnetic resonance scanners. Magn Reson Imaging 2000 Sep;18(7):8936.

(35) Glover GH, Law CS. Spiral-in/out BOLD fMRI for increased SNR and reduced susceptibility artifacts. Magn Reson Med 2001 Sep;46(3):515-22.

(36) Friedman L, Glover GH. Reducing interscanner variability of activation in a multicenter fMRI study: controlling for signal-to-fluctuation-noise-ratio (SFNR) differences. Neuroimage 2006 Nov 1;33(2):471-81.

(37) Glover GH, Lai S. Self-navigated spiral fMRI: interleaved versus single-shot. Magn Reson Med 1998 Mar;39(3):361-8.

(38) Boecker H, Kleinschmidt A, Requardt M, Hanicke W, Merboldt KD, Frahm J. Functional cooperativity of human cortical motor areas during self-paced simple finger movements. A high-resolution MRI study. Brain 1994 Dec;117 ( Pt 6):1231-9.

(39) Moritz CH, Meyerand ME, Cordes D, Haughton VM. Functional MR imaging activation after finger tapping has a shorter duration in the basal ganglia than in the sensorimotor cortex. AJNR Am J Neuroradiol 2000 Aug;21(7):1228-34.

(40) Moritz CH, Haughton VM, Cordes D, Quigley M, Meyerand ME. Whole-brain functional MR imaging activation from a finger-tapping task examined with independent component analysis. AJNR Am J Neuroradiol 2000 Oct;21(9):1629-35. 


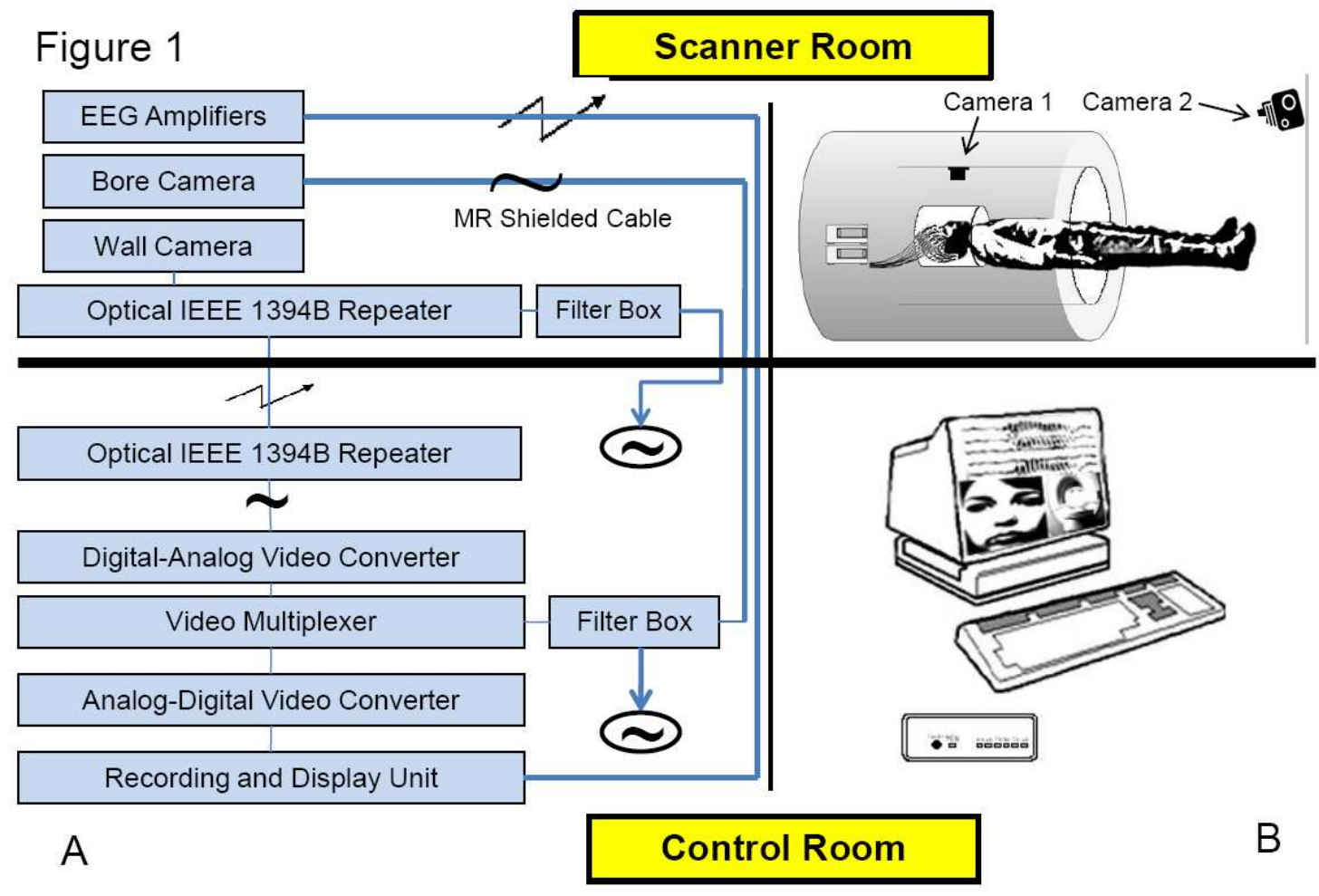


Figure 2

(A)

\begin{tabular}{|l|l|l|}
\hline Camera Position & RF ON & RF OFF \\
\hline C1 at P0, C2 at S0 & \\
\hline $\mathrm{C} 1$ at $\mathrm{P} 1, \mathrm{C} 2$ at $\mathrm{S} 1$ & \\
\hline $\mathrm{C} 1$ at $\mathrm{P2}, \mathrm{C} 2$ at $\mathrm{S} 1$ & & \\
\hline
\end{tabular}

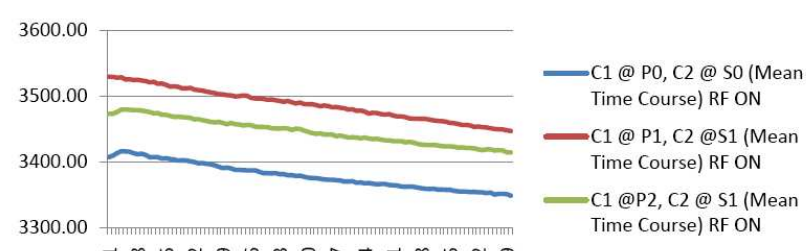

-
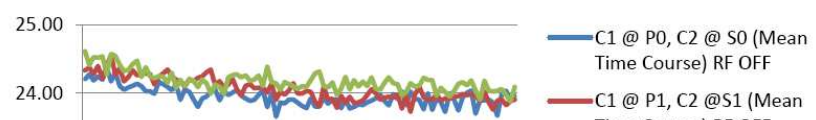

$\rightarrow \infty \sim$ ก

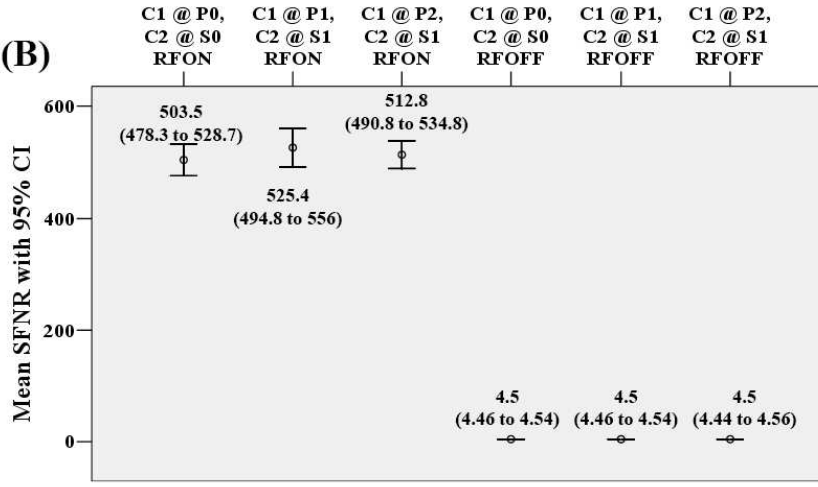

(C)

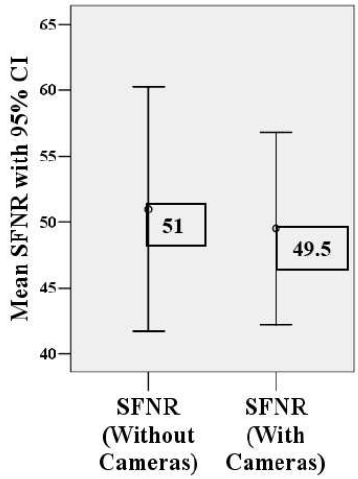


Figure 3

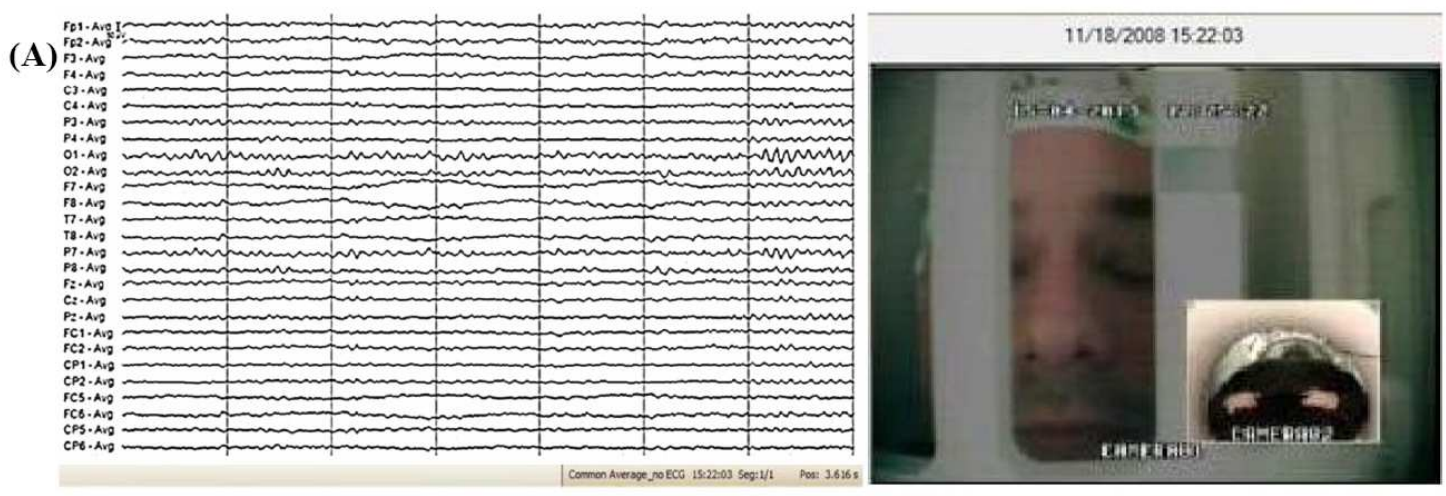

(B)

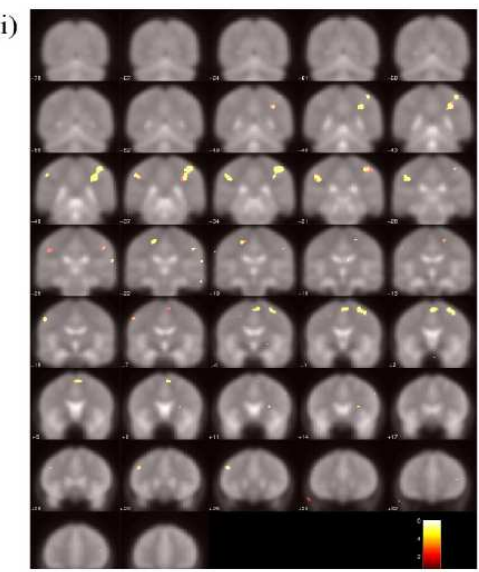

(ii)

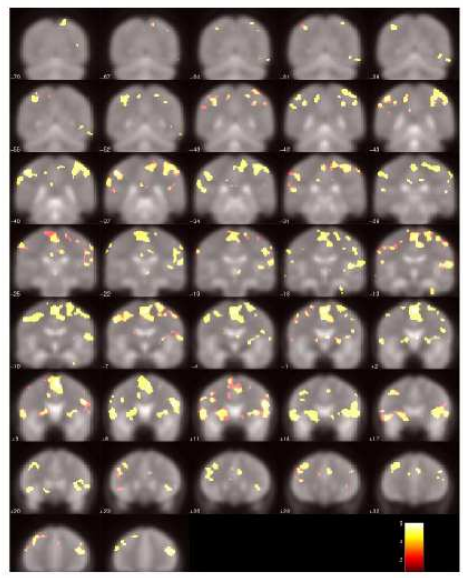

